\title{
Preventable medication harm across health care settings: a systematic review and meta-analysis
}

Alexander Hodkinson ${ }^{1 *}$, Natasha Tyler ${ }^{1,2}$, Darren M. Ashcroft ${ }^{2,3}$, Richard N. Keers 3,4, Kanza Khan ${ }^{3}$, Denham Phipps ${ }^{3}$, Aseel Abuzour ${ }^{3}$, Peter Bower ${ }^{1}$, Anthony Avery ${ }^{5}$, Stephen Campbell ${ }^{2}$ and Maria Panagioti ${ }^{1,2}$

\begin{abstract}
Background: Mitigating or reducing the risk of medication harm is a global policy priority. But evidence reflecting preventable medication harm in medical care and the factors that derive this harm remain unknown. Therefore, we aimed to quantify the prevalence, severity and type of preventable medication harm across medical care settings.

Methods: We performed a systematic review and meta-analysis of observational studies to compare the prevalence of preventable medication harm. Searches were carried out in Medline, Cochrane library, CINAHL, Embase and PsycINFO from 2000 to 27 January 2020. Data extraction and critical appraisal was undertaken by two independent reviewers. Random-effects meta-analysis was employed followed by univariable and multivariable meta-regression. Heterogeneity was quantified using the $l^{2}$ statistic, and publication bias was evaluated. PROSPERO: CRD42020164156.

Results: Of the 7780 articles, 81 studies involving 285,687 patients were included. The pooled prevalence for preventable medication harm was 3\% (95\% confidence interval (CI) 2 to $4 \%, P=99 \%)$ and for overall medication harm was 9\% (95\% $\mathrm{Cl} 7$ to $11 \%, P^{2}=99.5 \%$ ) of all patient incidence records. The highest rates of preventable medication harm were seen in elderly patient care settings (11\%, $95 \% 7$ to $15 \%, n=7)$, intensive care $(7 \%, 4$ to $12 \%, n=6)$, highly specialised or surgical care $(6 \%, 3$ to $11 \%, n=13)$ and emergency medicine $(5 \%, 2$ to $12 \%, n=12)$. The proportion of mild preventable medication harm was 39\% ( 28 to $51 \%, n=20, P=96.4 \%$ ), moderate preventable harm $40 \%$ (31 to $49 \%, n=22, P=93.6 \%$ ) and clinically severe or life-threatening preventable harm $26 \%$ ( 15 to $\left.37 \%, n=28, P^{2}=97 \%\right)$. The source of the highest prevalence rates of preventable harm were at the prescribing (58\%, 42 to $\left.73 \%, n=9, P^{2}=94 \%\right)$ and monitoring (47\%, 21 to $73 \%, n=8, P^{2}=99 \%$ ) stages of medication use. Preventable harm was greatest in medicines affecting the 'central nervous system' and 'cardiovascular system'.

(Continued on next page)
\end{abstract}

* Correspondence: alexander.hodkinson@manchester.ac.uk

${ }^{1}$ National Institute for Health Research School for Primary Care Research,

Centre for Primary Care and Health Services Research, Division of Population

Health, Health Services Research and Primary Care, School of Health

Sciences, Faculty of Biology, Medicine and Health, University of Manchester,

Manchester Academic Health Science Centre, Williamson Building, Oxford

Road, Manchester M13 9PL, UK

Full list of author information is available at the end of the article

(c) The Author(s). 2020 Open Access This article is licensed under a Creative Commons Attribution 4.0 International License, which permits use, sharing, adaptation, distribution and reproduction in any medium or format, as long as you give appropriate credit to the original author(s) and the source, provide a link to the Creative Commons licence, and indicate if changes were made. The images or other third party material in this article are included in the article's Creative Commons licence, unless indicated otherwise in a credit line to the material. If material is not included in the article's Creative Commons licence and your intended use is not permitted by statutory regulation or exceeds the permitted use, you will need to obtain permission directly from the copyright holder. To view a copy of this licence, visit http://creativecommons.org/licenses/by/4.0/ The Creative Commons Public Domain Dedication waiver (http://creativecommons.org/publicdomain/zero/1.0/) applies to the data made available in this article, unless otherwise stated in a credit line to the data. 


\begin{abstract}
(Continued from previous page)
Conclusions: This is the largest meta-analysis to assess preventable medication harm. We conclude that around one in 30 patients are exposed to preventable medication harm in medical care, and more than a quarter of this harm is considered severe or life-threatening. Our results support the World Health Organisation's push for the detection and mitigation of medication-related harm as being a top priority, whilst highlighting other key potential targets for remedial intervention that should be a priority focus for future research.
\end{abstract}

Keywords: Patient safety, Preventable medication harm, Prevalence, Meta-analysis, Medication error

\section{Background}

The Institute of Medicine report 'To Err is Human: Building a Safer health System' helped generate the patient safety movement by reporting that up to 98,000 deaths were due to medication and at least some of them could have been preventable [1-3]. On March 2017, the World Health Organisation (WHO) launched a global initiative to develop approaches to reduce severe, preventable medication harm in all countries by $50 \%$ over the next 5 years [4]. Similarly, in May 2018, the Institute for Healthcare Improvement of the USA launched a multi-organisation initiative to create a national action plan for the prevention of harm in healthcare [5].

In the USA alone, medication errors are the third most common cause of death behind heart disease and cancer [6] accounting for injuries to approximately 1.3 million people annually $[7,8]$. Similarly, in the UK, it was estimated that there are 237 million medication errors in England over a year period $[9,10]$, and preventable adverse drug reactions were estimated to cost the National Health Service (NHS) £98.5 million per annum, consume 181,626 bed days, cause 712 deaths and contribute to 1708 deaths during initial hospitalisation.

It is important to underline that not all harms caused by medication errors are preventable. However, distinction between preventable and non-preventable harm is becoming increasingly important, because it provides an indication of where best to invest limited resources for healthcare improvement in order to have the greatest benefit. From a quality improvement perspective, measuring harm, particularly preventable harm, alongside medication error [11] is critical. This would help ensure efforts are more patient-centred; can target the system rather than the individual, with the aim of enhancing clinical outcomes; reduce punitive concerns associated with the reporting methodology; allow for analysis of unintended results and encourage learning from events to continually improve the processes for detecting preventable harm [12]. Practitioners should also supplement preventable medication harm assessment with the measurement of certain contributory factors such as the severity of harm, the source at which the harm occurred (i.e. prescribing, transcribing, dispensing, administering or monitoring) and classification of harm according to the 'five rights' (patient, drug, dose, route, and time). Each of these factors could significantly inform quality improvement efforts [13].

Due to the ongoing global challenges in this area, it is important that healthcare providers, researchers and policy makers have a better understanding about the current prevalence rates and nature of preventable medication harm. Therefore, we undertook a systematic review and meta-analysis to inform the identification of targets for improvement efforts on estimating the prevalence of preventable medication harm across medical care settings including hospitals, primary care and various specialties. We also examined the clinical severity of preventable medication harm, and the impact of the medication use, stage and high-risk medication groups by building on what is already known from previous research efforts [14-16].

\section{Methods}

This systematic review was conducted and reported in accordance with the Reporting Checklist for Metaanalyses of Observational Studies (MOOSE). The completed MOOSE checklist is available in Additional file 1: Table S1. The review protocol is registered in PROSPERO under the review number: CRD42020164156.

\section{Data sources and searches}

We searched the five electronic bibliographic databases from 2000 to 27 January 2020: Medline, Cochrane library, Embase and PsycINFO via Ovid and CINAHL via EBSCO. The searches were supplemented by checking conference abstracts and screening grey literature sources (WHOLIS, Google Scholar, SIGLE). We also identified eligible studies by checking references in existing systematic reviews in the area. The full search strategy is available in the Additional file 2: Table S2.

\section{Eligibility criteria}

We included observational studies (retrospective and prospective cohorts; cross-sectional studies) in any medical care setting (primary, secondary and tertiary care) published from January 2000 onwards in English language. This date was selected, because it coincides with the publication of landmark patient safety reports, the 
increase of patient safety research in volume and the assessment of preventability $[1,17,18]$. The primary outcome was the prevalence of preventable medicationrelated harm including [19] adverse drug events (ADEs) or adverse drug reactions (ADRs) whether they were acts of omission or commission, incorrect medication/dose/ timing, administration of a medication to a patient with a known allergy, inadequate monitoring or other errors. For inclusion in this study, the study authors clearly defined their inclusion criteria as being about ADEs or ADRs and assessment of preventability. We only included studies that provide amenable data for inclusion in meta-analysis. Studies reporting medication errors or non-adherence and that involved only patients with readmissions due to recurring medication harm were excluded.

\section{Study selection and data extraction}

The titles and abstracts of all identified citations as well as potentially eligible full texts were screened independently by two reviewers (AH, MP), using pre-defined criteria. Disagreements were settled through consultation with a third team member (RNK). For eligible studies, we used a pilot-tested extraction spreadsheet, to extract descriptive data on study characteristics (e.g. number and age of participants, research design, systems used for data collection, assessment and preventability) and quantitative outcomes (prevalence, severity, medical care setting, the medication group and stage of medication use of preventable medication harm). Two independent researchers (AH and NT) performed the data extraction with disagreements resolved by discussion within the wider team (MP, RNK, DMA).

\section{Risk of bias assessment}

The quality of the studies was evaluated by two independent reviewers (AH and NT) using the NewcastleOttawa scale for cross-sectional and cohort studies. This assessed the representativeness of the sample size, response rate, ascertainment of the exposure, control of confounding variables, assessment of preventability and timing and appropriate statistical analysis, which provided a score ranging from 0 (lowest grade) to 10 (highest grade). A higher grade indicated a lower risk of bias. For our analyses, studies scoring 7 or above were considered as low risk, whereas studies scoring below 7 were considered as high risk of bias.

\section{Data analysis}

Prevalence of preventable medication harm was pooled using random-effects models with the DerSimonian-Laird approach [20]. Secondary analysis looked at WHO priority areas including the healthcare system setting (i.e. general hospital/internal medicine; emergency department or
ICU; highly specialised or surgical care-which included hospitals with long-term acute care facilities for dealing with certain disease categories such as cardiac, oncology, or orthopaedic problems; care units for paediatric patients, including neonatal intensive care units; and elderly patients, including geriatric patients aged above 64 years), the severity (i.e. mild, moderate, severe/life-threatening), stage of medication (i.e. using the US Pharmacopeia five major steps [21]: prescribing, transcribing and documenting, dispensing, administering and monitoring), patient age distribution (new born (less than 3 years), adolescents ( 3 to 18 years), adults (19 to 69 years), elder ( 70 years or above)) based on mean or median and medication group according to the Anatomical Therapeutic Chemical (ATC) Classification System [22].

Unadjusted prevalence, incidence and standard errors for the study-specific estimates were recalculated, based on the information of crude numerators and denominators provided in the individual studies. To keep the effect of studies with extremely small prevalence estimates on the overall estimate to a minimum, the variance of the study-specific prevalence was stabilised with the Freeman-Tukey double arcsine transformation [23]. Sensitivity analyses were done using other transformations, a range of sample sizes and adjustments for betweenstudy variance $\left(\tau^{2}\right)$ [24-27].

Univariable and multivariable meta-regressions were performed to investigate possible sources of heterogeneity using the following variables: WHO region (North America, Europe and Asia), drug class, median sample size, age group (adolescent, adults or mixed), medical setting (same as healthcare system setting), assessment method (medical record review or survey/telephone/voluntary report), standard preventability assessment method (i.e. Hallas criteria [28] or Schumock and Thornton scale [29]) and severity classification system, length of study (less than 6 months or more), design (prospective cohorts, retrospect cohorts or crosssectional) and sensitivity analysis with studies at lower risk of bias.

Heterogeneity was assessed using the $\chi^{2}$ test on Cochrane's $Q$ statistic and quantified by calculating $I^{2}$ [30]. Values of $25 \%, 50 \%$ and $75 \%$ for $I^{2}$ represent low, medium and high heterogeneity [31]. The presence of publication bias was assessed by inspection of funnel plots, the Egger's test and the trim-and-fill method. All analyses were done using the 'meta' and 'metafor' packages of the statistical software R (v.3.6.3) [32]; specifically, the 'metaprop' function was used for pooling prevalence rates.

\section{Patient and public involvement}

Six patients who were members of our research advisory panel were involved in the development of our research 
questions, research protocol and selection of outcomes and they advised on the interpretation and dissemination of results.

\section{Results}

The searches revealed 7868 articles, and after reviewing the full text screening, 81 studies met the inclusion criteria (see PRISMA flow chart in Fig. 1). A list of the eligible studies is included in Additional file 3: Table S3.

\section{Characteristics of included studies}

Twenty-six of the studies (32\%) were conducted in Europe, 23 studies $(28 \%)$ in the USA, and the rest of the studies were mainly carried out in South East Asia, South America or the Middle East. The median sample size across studies was 1234 patients (IQR estimate 2652). The pooled sample size across the 81 eligible studies was 285,687 patients. Of these, 18,243 (6.4\%) patients experienced at least one classified medication related harm, and 7075 (2.5\%) experienced at least one preventable medication harm. In total, 20,698 medication harm incidents were reported, of which 7589 (37\%) were preventable medication harm.

Fifty-eight studies (72\%) used prospective cohort designs, $15(19 \%)$ retrospective cohort designs and eight (10\%) cross-sectional designs (Additional file 4: Table
S4). The most common setting of medical care was general hospitals or internal medicine settings in 29 studies (36\%). Fourteen (17\%) studies involved patients subject to highly specialised care (seven of which included patients in surgery [33-39] and two involving psychiatric patients [40, 41]), twelve (15\%) studies were situated in an emergency department, six $(10 \%)$ in an ICU and four (5\%) studies in primary care. The focus in nine $(11 \%)$ of the studies was on paediatric care units, with three of these studies being situated in neonatal intensive care units [42-44]. Seven $(9 \%)$ studies involved elderly patient care units, six of which were specialised care units for geriatric patients aged above 65 years [35, 45-49].

Preventable medication harm was assessed in 54 (67\%) studies by use of medical record reviews or observations. In contrast, 27 (33\%) studies reported preventable harm by survey, telephone or spontaneous reporting surveillance systems. At least one of the standard methods [28, 29] for assessing the preventability of medication harm involving consensus between two or more trained reviewers (physicians or nurses) was applied in 48 (59\%) studies. However, 20 (25\%) of the studies used adapted scales where consensus procedures were not well known, and 13 studies (16\%) had not defined the method used for assessment of preventability.

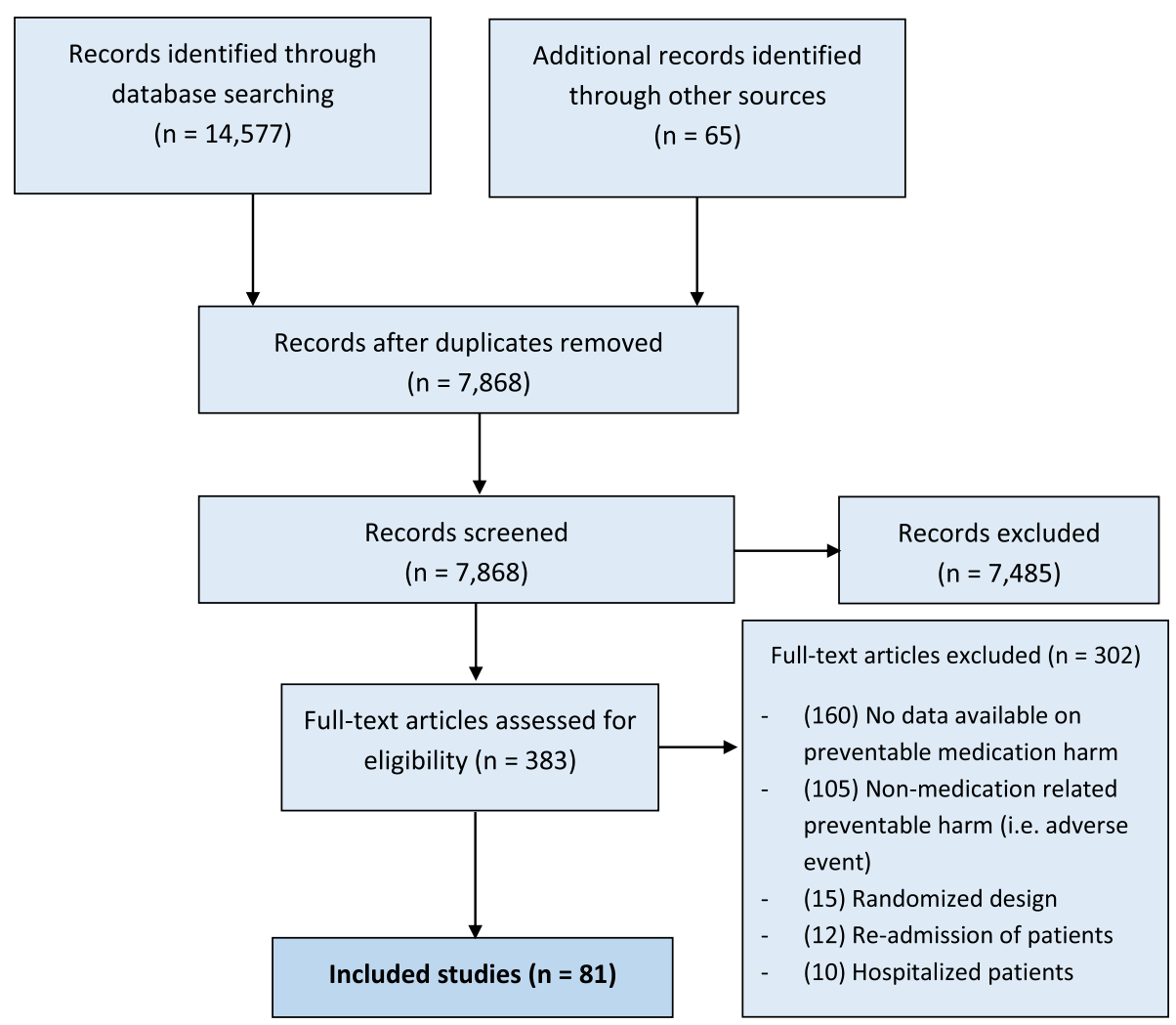

Fig. 1 PRISMA flowchart of the included studies 


\section{Quality assessment}

The median Newcastle-Ottawa score for included studies was 7 (range 6 to 9). Thirty-five studies (43\%) scored eight or above and were considered to be at low risk of bias (see full assessment in Additional file 5: Table S5).

\section{Pooled prevalence rates of medication-related harm}

Table 1 shows that the unadjusted pooled prevalence of preventable medication harm was 3\% (95\% CI 2 to $4 \%$, $\left.I^{2}=99 \%\right)$ and the pooled prevalence of all medication harm (inclusive of non-preventable) was 9\% (95\% CI 7 to $\left.11 \%, I^{2}=99.5 \%\right)$.

The highest prevalence rates for preventable medication harm were observed in elderly patient care units $(11 \%, 95 \%$ CI 7 to $15 \%, n=7))$, and patients in ICUs $(7 \%, 4$ to $12 \%, n=6)$ ), highly specialised or surgical care $(6 \%, 3$ to $11 \%, n=13))$ and emergency departments $(5 \%$, 2 to $12 \%, n=12)$ ) (Fig. 2). In contrast, the lowest prevalence rates were observed in paediatric care units $(1 \%, 0$ to $2 \%, n=9)$, primary care $(1 \%, 1$ to $2 \%, n=4)$ and general hospitals or internal medicine settings (2\%, 1 to $2 \%$, $n=29$ ). Prevalence rates by patient age group are provided in Fig. 3. These results re-enforce earlier findings, showing greater preventable medication harms in elderly patients $(4 \%, 2$ to $8 \%, n=16)$ and the lowest rate of preventable medication harm in paediatric patients $(1 \%, 0$ to $2 \%, n=9)$.

\section{Clinical severity, stage of medication use and medication group}

As shown in Table 1, the proportions of clinical severity for mild preventable medication harm was 39\% (95\% CI 28 to $51 \%, n=20, I^{2}=96.4 \%$ ), moderate preventable harm was $40 \%$ (31 to $49 \%, n=22, I^{2}=93.6 \%$ ) and severe or potentially life-threatening preventable harm was $26 \%$ (15 to $37 \%, n=28, I^{2}=97 \%$ ).

The highest rates of preventable medication harm were sourced at the prescribing $(58 \%, 95 \% 42$ to $73 \%$, $\left.n=9, I^{2}=94 \%\right)$ and monitoring $(47 \%, 95 \% 21$ to $73 \%$, $\left.n=8, I^{2}=99 \%\right)$ stages. The stages with the lowest rates of preventable medication harm were sourced at dispensing $\left(2 \%, 95 \% 0\right.$ to $\left.6 \%, n=6, I^{2}=47 \%\right)$ and transcribing/documenting ( $3 \%, 95 \% 0$ to $9 \%, n=8, I^{2}=78 \%$ ) stages.

Table 1 Proportions of the different types of preventable medication harm and overall medication harm

\begin{tabular}{|c|c|c|c|c|c|c|c|}
\hline \multirow[t]{2}{*}{ Outcome } & \multirow[b]{2}{*}{$\begin{array}{l}\text { Number of } \\
\text { studies }\end{array}$} & \multicolumn{3}{|c|}{ Preventable medication harm } & \multicolumn{3}{|c|}{ Any medication harm } \\
\hline & & $\begin{array}{l}\text { Prevalence rate, \% } \\
(95 \% \mathrm{Cl}) *\end{array}$ & $I^{2}, \%$ & $\begin{array}{l}\text { Median } \\
\text { (IQR) }\end{array}$ & $\begin{array}{l}\text { Prevalence rate, \% } \\
(95 \% \mathrm{CI}) *\end{array}$ & $I^{2}, \%$ & $\begin{array}{l}\text { Median } \\
\text { (IQR) }\end{array}$ \\
\hline Overall prevalence rate & 81 & $3(2,4)$ & 99 & $2.9(0.02-27.1)$ & $9(7,11)$ & 99.5 & $9.8(0.24-65.4)$ \\
\hline \multicolumn{8}{|l|}{ Healthcare system: } \\
\hline $\begin{array}{l}\text { - General hospital } \\
\text { or internal medicine }\end{array}$ & 29 & $2(1,2)$ & 98.4 & $1.6(1.2-2.2)$ & $5(4,7)$ & 99.0 & $5.1(3.2-10.9)$ \\
\hline - Emergency department & 12 & $5(2,12)$ & 99.7 & $5.2(2.1-12)$ & $9.3(4,21)$ & 99.8 & $6.2(6.0-14)$ \\
\hline$-I C U$ & 6 & $7(4,12)$ & 91.1 & $6.8(3.9-11.6)$ & $14(8,23)$ & 95.6 & $16.4(9.5-20.2)$ \\
\hline $\begin{array}{l}\text { - Highly specialised } \\
\text { care or surgical }\end{array}$ & 13 & $6(3,11)$ & 98.7 & $5.4(3.0-9.7)$ & $18(11,26)$ & 99.3 & $22.9(17.8-26.2)$ \\
\hline - Paediatric & 9 & $1(0,2)$ & 94.9 & $1.1(0.1-2.1)$ & $5(2,10)$ & 99.2 & $5(2.4-14.3)$ \\
\hline - Elderly & 7 & $11(7,15)$ & 95.9 & $10.7(7.2-15.4)$ & $23(13,37)$ & 99.1 & $24.8(16-31.3)$ \\
\hline - Primary care & 4 & $1(1,2)$ & 84.9 & $1.2(1-1.9)$ & $10(6,16)$ & 99.0 & $9.9(8-13.9)$ \\
\hline - Psychiatric & 1 & $1(1,2)$ & NA & NA & $10(9,12)$ & NA & NA \\
\hline \multicolumn{8}{|l|}{ Severity of preventable harm: } \\
\hline - Mild & NA & $39(28,51), n=20$ & 96.4 & $40(21-49)$ & $50(44,56), n=47$ & 98 & $46.9(35.9-63.1)$ \\
\hline - Moderate & NA & $40(31,49), n=22$ & 93.6 & $36.5(28.1-54.4)$ & $41(36,47), n=54$ & 98 & $37.3(24.7-53.3)$ \\
\hline - Severe/life-threatening & NA & $26(15,37), n=28$ & 97 & $19.5(8-40)$ & $14(10,19), n=55$ & 98 & $10(6.4-18.6)$ \\
\hline \multicolumn{8}{|l|}{ Stages of medication use $e^{\beta}$ : } \\
\hline 1. Prescribing & 9 & $58(42,73), n=9$ & 94 & $55.8(35.4-77.3)$ & $64(34,84), n=18$ & 98.5 & $52(36-79)$ \\
\hline 2. Transcribing/documenting & 8 & $3(0,9), n=8$ & 78 & $0.9(0-6.3)$ & $6(1,14), n=17$ & 89 & $2(0.5-9)$ \\
\hline 3. Dispensing & 6 & $2(0,6), n=6$ & 47 & $3.8(0.9-4.1)$ & $8(2,24), n=15$ & $69 \%$ & $5.6(1.3-8)$ \\
\hline 4. Administering & 15 & $21(11,33), n=15$ & 93 & $20.8(8.8-31.1)$ & $25(12,40), n=32$ & 96.8 & $19(7.6-30)$ \\
\hline 5. Monitoring & 8 & $47(21,73), n=8$ & 99 & $54.9(25.3-76)$ & $54(16,82), n=15$ & 100 & $35(15-70)$ \\
\hline
\end{tabular}

*Freeman-Tukey double arcsine transformation was used for the prevalence rate calculations

${ }^{\beta}$ Stages of medication use follow the using the USA's Pharmacopeia 5 major steps 


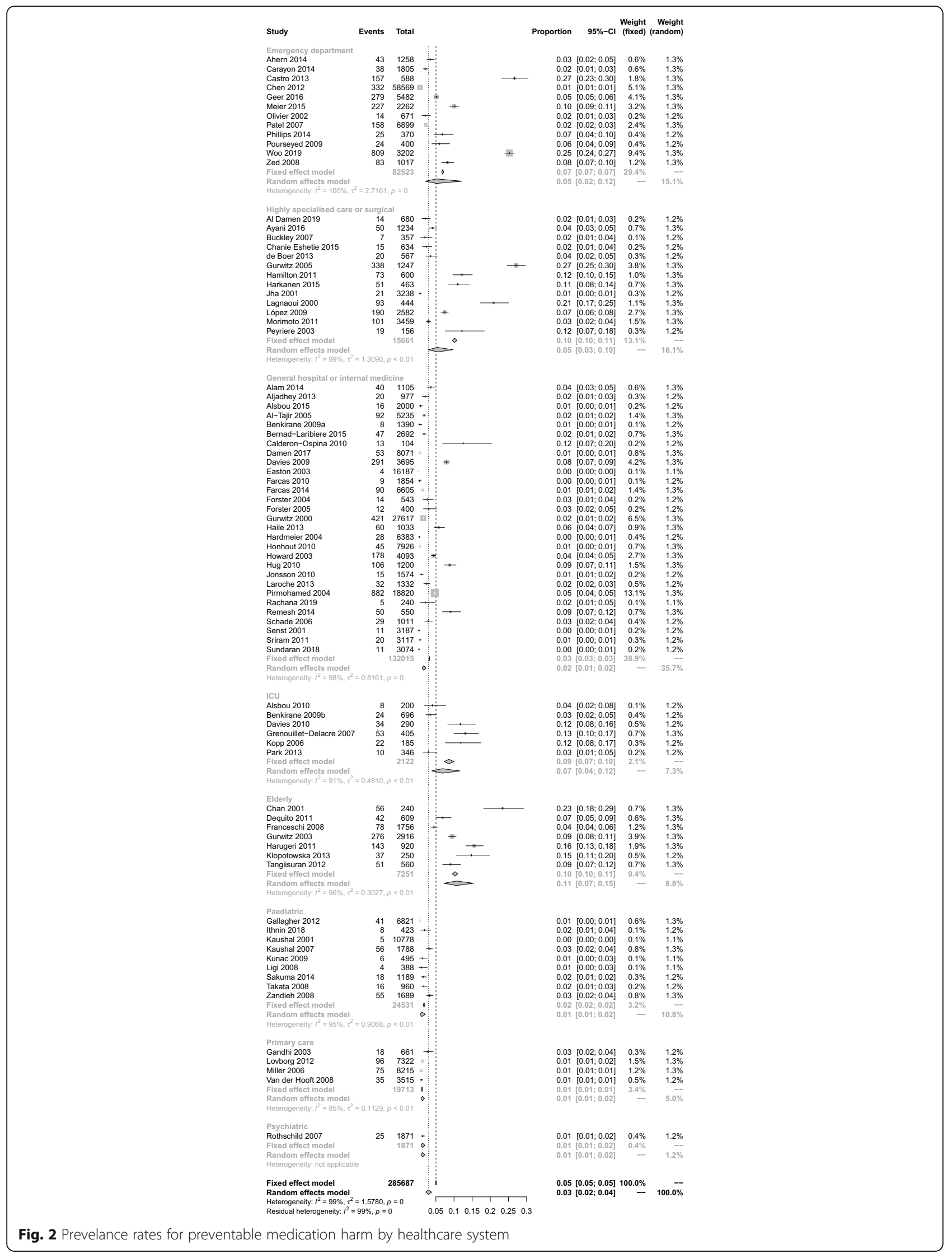




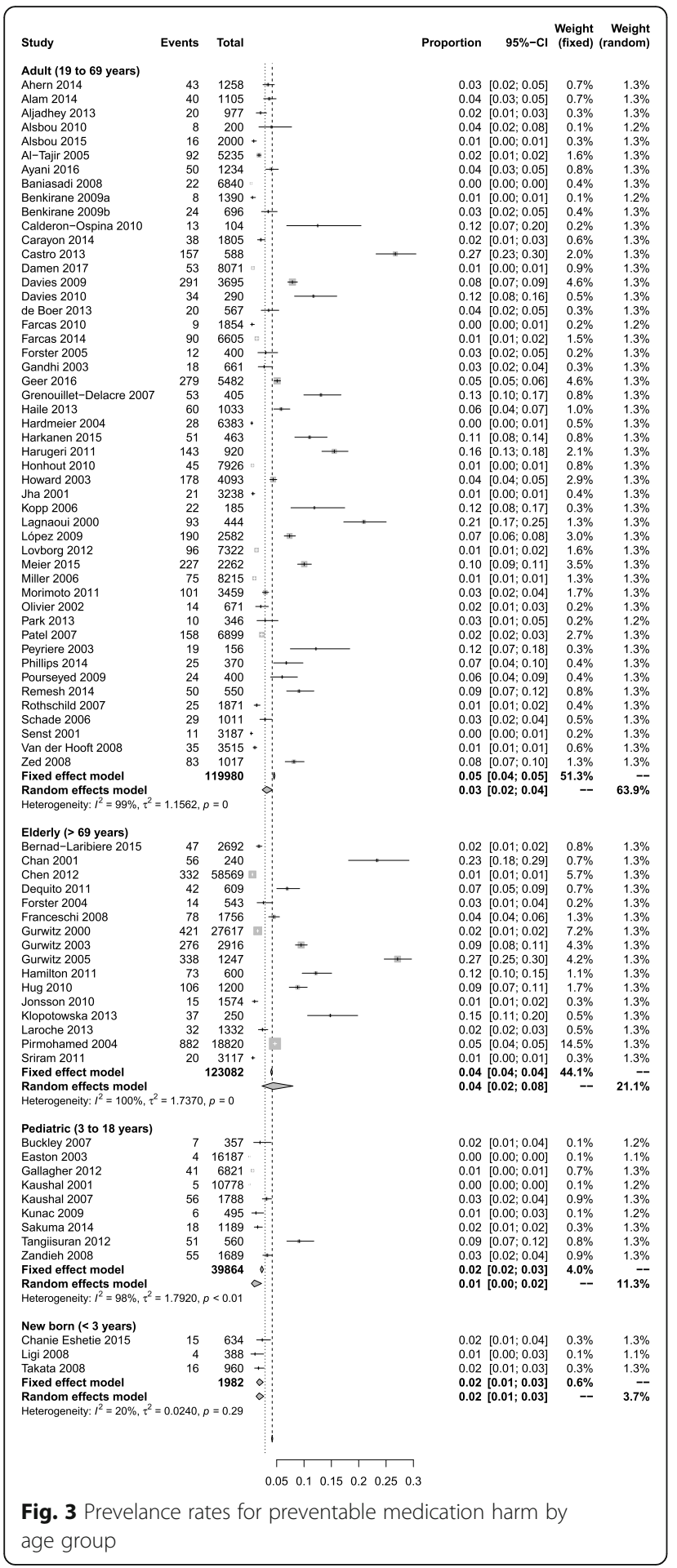

Preventable harm was greatest in medicines affecting the 'central nervous system' (ATC code N) (21\%, 95\% 6 to $43 \%, n=6$ ) (see Table 2). However, prevalence rates were also high in the ATC medication groups for 'cardiovascular' (code C) $(16 \%, 95 \% 11$ to $23 \%, n=14)$, 'hypnotics and sedatives' (code N05C) (16\%, 95\% 11 to $21 \%$, $n=5)$, 'anti-inflammatory and antirheumatic products' (code M01) (15\%, 95\% 7 to $26 \%, n=5)$ and 'antibiotics and antibacterial for systemic use' (code J01) (12\%, 95\% 7 to $18 \%, n=12$ ). The prevalence rate was below $5 \%$ for patients receiving 'respiratory system-related drugs' (code R), drugs for patients with 'functional gastrointestinal disorders' (code A03), patients taking 'corticosteroids for systemic use' (code H02) and 'antiepileptics' (code N03). The top five medication groups associated with preventable medication harm in each of the 20 studies is provided in Additional file 6: Table. S6.

\section{Meta-regressions to explore heterogeneity in the prevalence of preventable medication harm}

Table 3 shows the results of the univariable and multivariable analysis. The univariable analyses showed that the prevalence of preventable medication harm was higher across studies with smaller ( $\leq 1200$ patients) sample sizes $(b=0.111,95 \%$ confidence interval 0.064 to $0.159)$, those carried out in Europe $(b=0.031,-0.0002$ to 0.061$)$ and at lower risk of bias $(b=0.075,0.022$ to 0.128 ). The prevalence of preventable harm was lower in children or adolescent-based studies $(b=-0.088,-0.151$ to -0.025$)$ and in studies that assessed medication harm by survey, telephone or spontaneous reporting surveillance systems $(b=-0.026,-0.053$ to 0.0003$)$. When compared to general hospitals/internal medicine, healthcare settings involving the elderly $(b=0.094,0.052$ to 0.136), emergency departments $(b=0.042,0.011$ to $0.073)$, highly specialised or surgical care $(b=0.062$, 0.030 to 0.093$)$ or intensive care $(b=0.052,0.010$ to 0.094) showed elevated significant levels of preventable harm. These five variables (sample size, healthcare setting, WHO region, age group, assessment method and low risk of bias) were therefore eligible for inclusion in the multivariable regression analysis.

The multivariable model was statistically significant $\left(\chi^{2}\right.$ (6) $\left.=44.228, P<0.001, R^{2}=37.65 \%\right)$ and reduced the $I^{2}$ statistic from 99 to $61.3 \%$. Only two of the variables remained statistically significant; studies with low sample sizes $(b=0.038,0.016$ to 0.060$)$ and the three healthcare setting groups, including elderly patient care $(b=0.07$, 0.023 to 0.107$)$, emergency medicine $(b=0.028,-0.002$ to 0.058$)$ and highly specialised care $(b=0.052,0.022$ to 0.082 ), were all associated with a higher prevalence of preventable medication harm.

\section{Publication bias}

Additional file 7: Fig. S1 shows some evidence of publication bias as indicated by visual inspections of the funnel plots and by the Egger regression test for small study effects for the primary outcome (bias coefficient for the main analysis $1.39,95 \% 0.38$ to $3.47, P<0.001$ ). Trimand-fill method also revealed evidence of publication bias. 
Table 2 Proportions of preventable medication harm by medication class

\begin{tabular}{|c|c|c|c|c|c|c|}
\hline Drug class & ATC code & $N$ studies & Prevalence, \% & $95 \% \mathrm{Cl}$ & $\tau^{2}$ & $I^{2}(\%)$ \\
\hline Analgesics & $\mathrm{NO} 2^{¥}$ & 4 & 7 & $0.5,19$ & 0.0229 & 84.00 \\
\hline Antibiotics and antibacterial & $\mathrm{J} 01^{*}$ & 12 & 12 & 7,18 & 0.0165 & 87.80 \\
\hline Antithrombotic agents/anticoagulants & $\mathrm{B} 01^{*}$ & 10 & 11 & 7,15 & 0.0073 & 78.60 \\
\hline Antidepressants & $N 06 A^{\beta}$ & 4 & 6 & $0.4,17$ & 0.0253 & 94.90 \\
\hline Antidiabetic & $\mathrm{A} 10^{*}$ & 4 & 7 & $0.8,17$ & 0.0162 & 77.50 \\
\hline Antiepileptics & $\mathrm{N}^{*} 3^{*}$ & 3 & 4 & $0.2,10$ & 0.0029 & 26.40 \\
\hline Anti-inflammatory and antirheumatic & ${\mathrm{M} 01^{*}}^{*}$ & 5 & 15 & 7,26 & 0.0166 & 79.40 \\
\hline Antipsychotics & N05 $A^{\beta}$ & 4 & 11 & $0.2,32$ & 0.0622 & 95.60 \\
\hline Cardiovascular & $C^{*}$ & 14 & 16 & 11,23 & 0.0205 & 90.80 \\
\hline Corticosteroids & $\mathrm{H} 02^{*}$ & 5 & 2 & $0.2,6$ & 0.0066 & 77.10 \\
\hline Diuretics & $\mathrm{CO}^{*}$ & 5 & 6 & $0.2,17$ & 0.0365 & 95.80 \\
\hline Functional gastrointestinal disorders & $\mathrm{A}_{0} 3^{*}$ & 3 & 4 & 0,14 & 0.0183 & 75.80 \\
\hline Musculoskeletal system & $M^{*}$ & 3 & 9 & $2.4,20$ & 0.0142 & 85.00 \\
\hline Nervous system & $N^{*}$ & 6 & 21 & 6,43 & 0.0784 & 97.00 \\
\hline Opioids & $\mathrm{N} 02 \mathrm{~A}^{\beta}$ & 5 & 6 & 2,10 & 0.0045 & 74.70 \\
\hline Respiratory system-related drugs & $\mathrm{R}^{*}$ & 4 & 1 & 0,4 & 0.0061 & 74.90 \\
\hline Sedatives & N05C $C^{\beta}$ & 5 & 16 & 11,21 & 0.0016 & 33.50 \\
\hline
\end{tabular}

*ATC 1st level-related to mostly body function;

${ }^{¥}$ ATC 2nd level-pharmacological or therapeutic subgroup

${ }^{\beta}$ ATC 3rd and 4th levels-chemical, pharmacological or therapeutic subgroup

\section{Discussion}

This meta-analysis found that preventable medication harm occurs in $3 \%$ of patients across medical care settings and that at least a quarter of preventable medication harm is severe or potentially life threatening. In our previous meta-analysis, the prevalence of preventable patient harm (e.g. adverse events due to any type medical errors-not only medication) was $6 \%$, and only one tenth of this harm was severe or potentially life-threatening [50]. Thus, medication harm indeed accounts for half of the overall preventable harm in medical care. The present findings also align closely to a recent narrative meta-review of systematic reviews [51], which looked at the preventability of ADRs in patients receiving acute or ambulatory care-reporting a prevalence rate of $3.13 \%$. However, this earlier narrative overview involved only 37 studies compared to the 81 studies in our meta-analysis, did not include patients in primary care or emergency care settings and did not examine thoroughly the severity of harm, patient age, stage of medication use and high-risk medication groups.

The highest prevalence rate of preventable medication harm was seen in studies based on elderly care units which often involve patients with high comorbidity and hence polypharmacy. The headline policy implication of this study therefore is that mitigation strategies for preventable medication harm are primarily needed for older people with multimorbidity/ polypharmacy-which is indeed a key priority area of the WHO safety initiative [16, 52]. A recent review that tried to determine which interventions, alone or in combination, were effective in improving the appropriate use of polypharmacy and reducing medication related harms in older people [53], revealed great uncertainty about the effectiveness of comprehensive medicines reviews of patient prescriptions and urged for further research. Since the last update of this review, a Scottish working group has published a guidance document on polypharmacy, which included a seven-step process for standardised and structured medicines reviews that are holistic, patient-centred and consider non-pharmacological treatments [54], as well as a review of the quality of development of available guidelines to promote appropriate polypharmacy [55]. Further population-based research is needed to evaluate the implementation and effect of these resources on prescribing for older people.

Moreover, medication harm in ICUs [56] and acute specialised care settings involving surgery [57] was associate with higher volumes of preventable medication harm and should, therefore, also be considered as highrisk patient care settings. Evidence concerning the prevalence and severity of preventable medication harm in primary care and psychiatry was scarce. We only found four studies based in primary care, where over $80 \%$ of healthcare services are delivered internationally [58], and only one study was identified in psychiatry. It is therefore possible that certain types of preventable harm in 
Table 3 Univariable and multivariable meta-regression analysis of prevalence estimation for preventable medication harm

\begin{tabular}{|c|c|c|c|c|c|c|c|}
\hline \multirow[b]{2}{*}{ Variable } & \multirow[b]{2}{*}{ No. } & \multicolumn{3}{|l|}{ Univariable } & \multicolumn{3}{|l|}{ Multivariable } \\
\hline & & $\begin{array}{l}\text { Regression coefficient } \\
(95 \% \mathrm{Cl})\end{array}$ & SE & $P$ value & $\begin{array}{l}\text { Regression coefficient } \\
(95 \% \mathrm{Cl})\end{array}$ & SE & $P$ value \\
\hline \multicolumn{8}{|l|}{ WHO region: } \\
\hline - Asia/other & 32 & 1 & - & - & 1 & - & - \\
\hline - North America & 23 & $0.018(-0.014,0.049)$ & 0.016 & 0.273 & $0.03(-0.02,0.087)$ & 0.028 & 0.237 \\
\hline - Europe & 26 & $0.031(-0.0002,0.061)$ & 0.016 & 0.051 & $0.011(-0.045,0.066)$ & 0.028 & 0.709 \\
\hline \multicolumn{8}{|l|}{ Drug classification: } \\
\hline - Yes & 67 & 1 & - & - & - & - & - \\
\hline - No & 14 & $-0.011(-0.081,0.059)$ & 0.036 & 0.760 & NA & NA & NA \\
\hline \multicolumn{8}{|l|}{ Sample size*: } \\
\hline - > 1,200 patients & 41 & 1 & - & - & 1 & - & - \\
\hline - $\leq 1,200$ patients & 40 & $0.111(0.064,0.159)$ & 0.024 & $<0.0001$ & $0.038(0.016,0.060)$ & 0.011 & 0.036 \\
\hline \multicolumn{8}{|l|}{ Age group: } \\
\hline - Adults & 63 & 1 & - & - & 1 & - & - \\
\hline - Children/adolescents & 18 & $-0.088(-0.151,-0.025)$ & 0.032 & 0.0060 & $-0.016(-0.048,0.016)$ & 0.016 & 0.319 \\
\hline \multicolumn{8}{|l|}{ Healthcare setting: } \\
\hline - General hospital or internal medicine & 29 & 1 & - & - & 1 & - & - \\
\hline - Elderly & 7 & $0.094(0.052,0.136)$ & 0.021 & $<0.0001$ & $0.07(0.023,0.107)$ & 0.021 & 0.002 \\
\hline - Emergency department & 12 & $0.042(0.011,0.073)$ & 0.016 & 0.009 & $0.028(-0.002,0.058)$ & 0.015 & 0.062 \\
\hline - Highly specialised care or surgical & 13 & $0.062(0.030,0.093)$ & 0.016 & 0.0001 & $0.052(0.022,0.082)$ & 0.015 & 0.0007 \\
\hline$-I C U$ & 6 & $0.052(0.010,0.094)$ & 0.021 & 0.015 & $0.038(-0.004,0.080)$ & 0.021 & 0.073 \\
\hline - Paediatric & 9 & $-0.008(-0.044,0.028)$ & 0.018 & 0.663 & $-0.0001(-0.044,0.043)$ & 0.022 & 0.996 \\
\hline - Primary care & 4 & $-0.009(-0.056,0.039)$ & 0.024 & 0.720 & $-0.014(-0.058,0.031)$ & 0.023 & 0.547 \\
\hline - Psychiatric & 1 & $-0.010(-0.100,0.080)$ & 0.046 & 0.825 & $-0.006(-0.090,0.079)$ & 0.043 & 0.897 \\
\hline \multicolumn{8}{|l|}{ Assessment method: } \\
\hline $\begin{array}{l}\text { - Medical record review/chart review } \\
\text { or observation }\end{array}$ & 54 & 1 & - & - & 1 & - & - \\
\hline $\begin{array}{l}\text { - Survey, telephone, voluntary } \\
\text { (spontaneous) report }\end{array}$ & 27 & $-0.026(-0.053,0.0003)$ & 0.014 & 0.053 & $-0.021(-0.045,0.004)$ & 0.012 & 0.099 \\
\hline \multicolumn{8}{|l|}{ Standard method for preventability: } \\
\hline - Yes & 49 & 1 & - & - & NA & NA & NA \\
\hline$-\mathrm{No}$ & 32 & $-0.014(-0.069,0.040)$ & 0.028 & 0.613 & NA & NA & NA \\
\hline \multicolumn{8}{|l|}{ Length of study: } \\
\hline$-\leq 6$ months & 47 & 1 & - & - & NA & NA & NA \\
\hline - > 6 months & 34 & $-0.026(-0.080,0.029)$ & 0.028 & 0.357 & NA & NA & NA \\
\hline \multicolumn{8}{|l|}{ Design: } \\
\hline - Prospective & 58 & 1 & - & - & - & - & - \\
\hline - Retrospective cohort or cross-sectional & 23 & $0.011(-0.019,0.040)$ & 0.015 & 0.480 & NA & NA & NA \\
\hline \multicolumn{8}{|l|}{ Risk of bias: } \\
\hline - High (score < 7) & 31 & 1 & - & - & NA & NA & NA \\
\hline - Low (score $\geq 7$ ) & 50 & $0.075(0.022,0.128)$ & 0.027 & 0.006 & $0.019(-0.004,0.043)$ & 0.012 & 0.107 \\
\hline \multicolumn{5}{|l|}{ Model fit indices } & \multicolumn{3}{|c|}{$x^{2}(6)=44.228, P<0.001, R^{2}=37.65 \%$} \\
\hline
\end{tabular}


psychiatry settings may remain undetected, as these harms often result from multiple interacting errors from violation provoking conditions and latent 'system' failures $[22,59]$. Thus, there is a need for more research in both these care settings.

The prescribing and monitoring stages of medication use were key sources of preventable harm. The adoption of electronic health records and electronic prescribing has helped avert preventable harm at the prescribing and transcribing stages [14, 60-66], but preventable harm persists across all pathways of medication use. This is most likely due to underlying system flaws that allow individual errors such as those in prescribing or medicines administration to reach the patient and cause serious harm [67]. Human factors play an important role in understanding these system flaws; for instance, there may be a lack of standard procedures for storage of medications that look alike, poor communication between different providers, lack of verification before medication administration and limited involvement of patients in their own care [68-71]. Better safeguarding processes that acknowledge the contribution of human factors and systems thinking are required at the different points of medication use to ensure correct measures are contributing to healthcare improvement efforts [72].

We found that medication groups that gave rise to most preventable medication harm include; central nervous system, cardiovascular, hypnotics and sedatives, anti-inflammatory and antirheumatic, antibiotics and antibacterial drugs. Many of these medication groups have been previously assessed in relation to hazardous prescribing in the past $[14,15]$. However, the interventions featured in these studies deliberately focused on potential medication errors rather than preventable harm, and therefore, available evidence has not yet determined whether these interventions will reduce harm to patients. This stresses further the urgency and need to focus on assessment of medication errors and harm in conjunction [13, 71], as well as new ways of reducing medication harms in older people [53].

Most efforts so far originate in developed countries [73], and so the rate of preventable medication harm from less developed countries remains relatively unknown. However, in one study, the preventability of adverse events across 26 hospitals in eight low-and middleincome countries showed an adverse event rate to be around $8 \%$. Of these events, $83 \%$ were preventable, while about $30 \%$ were associated with death of the patient [74]. Although this is likely to be an underestimate with higher rates of excess death unaccounted for, it shows that patient safety is a much bigger problem in these developing and transitional countries, when judged by the preventability and severity of harm (number of preventable deaths). These results are somewhat contrasting with our results, with the higher rate of preventable harm being found in studies from Europe. However, these findings might reflect the limitations that exist in assessing preventable medication harm in developing countries. Moreover, the prevention of medication harm is often complex and involves improving basic clinical processes and does not simply depend on the provision of more resources [75].

Despite this being the first large meta-analysis to assess the prevalence of preventable medication harm across healthcare settings, there still remain several limitations. First, a considerable proportion of the high heterogeneity remained unexplained in meta-regressions. Several other factors such as differences in systems, procedures and variations in timeframe used to evaluate medication harm remain largely unknown and may be responsible for this unexplained heterogeneity.

Second, whilst we performed exhaustive searches for unpublished studies, the exclusion of non-English written studies and the presence of publication bias that forms of selection bias or system bias is likely [76]. We tried to account for this in the analysis by adjusting for sample size, but some of the causal factors remain unexplained.

Third, studies not reporting data on preventable medication harm were excluded from the analyses. Among the included studies, only $36 \%$ of studies provided an analysis of severity and even the classification system used was unclear at times so a pragmatic judgement was made to group some of the severity categories. Furthermore, discussions of causality assessment were limited with few studies reporting the exact assessment tool used, and only $22 \%$ of studies provided the stage of medication use, and whilst harm was reported by medication group in $78 \%$ of the studies, only one fifth of these provided data at the preventable level. The use of different preventability scales may also incur a level of 'hindsight bias', where healthcare professionals may be subject to overestimation of their ability to predict preventable harm events [77, 78]. However, adjustment for this in observation research can be a significant challenge in evidence synthesis.

Finally, more than two thirds of preventable medication harm were examined retrospectively through medical case notes and chart reviews. Although case note reviews are the most universally used method for assessing medication harm to date, patients and healthcare providers suggest that case reviews still lack the robustness to detect diagnostic error and are susceptible to time-delay issues in the absence of regular patient consultation [79]. Combining methods that prospectively uncover preventable harm by use of the 'failure mode and effect analysis', 'structured what-if technique' [13], pharmacist screening, or patient surveys along with 
retrospective error detection methods including trigger tools, voluntary reporting systems, root cause analysis or mortality reviews [76, 80] would provide better and more promising approaches for enhancing the detection of preventable medication harm.

\section{Conclusions}

Our study findings confirm that preventable medicationrelated harm is a frequent and enduring serious problem, causing severe or potentially life-threatening outcomes in over a quarter of all preventable harm cases. A highly problematic healthcare setting was in geriatric care, specialised care settings, intensive care or those in emergency departments. Elderly patients are at greater risk of polypharmacy, and patients in other specialised or emergency medicine settings may be exposed to higher-risk mediation groups. As both are priority areas of the WHO's safety initiative, we have gone some way in this review to providing a basis of evidence to support future policy developments in this area. Nevertheless, further research is needed in primary care and psychiatry settings where up to $80 \%$ of healthcare service is delivered, alongside efforts to better understand where improvements can be made in less-developed countries.

\section{Supplementary information}

Supplementary information accompanies this paper at https://doi.org/10 1186/s12916-020-01774-9.

\footnotetext{
Additional file 1: Table S1. Moose checklist.

Additional file 2: Table S2. Searches.

Additional file 3: Table S3. Citations for eligible studies included in meta-analysis.
}

Additional file 4: Table S4. Characteristics of included studies. Additional file 5: Table S5. Critical appraisal ratings for all studies included in review.

Additional file 6: Table S6. Top five most common preventable medication harms by ATC drug classification. \% represent prevalence's.

Additional file 7: Figure S1. Funnel plot of preventable medication harm (log-transformed proportion).

Acknowledgements

None to declare

\section{Consent to participate}

Not applicable

\section{Disclaimer}

The views expressed are those of the author(s) and not necessarily those of the UK General Medical Council, the NIHR Greater Manchester Patient Safety Translational Research Centre or the Department of Health and Social Care.

\section{Authors' contributions}

MP, DMA, RNK and AJA had the initial research idea and obtained funding for this study. AH, MP, DMA and RNK formulated the research questions and designed the study. AH, MP and KK searched for published work, selected articles, extracted and analysed data, and drafted the protocol and manuscript. NT, RNK and DMA helped in the data selection and NT in the data extraction. MP and NT substantially contributed in designing the searches and the statistical analysis plan, writing of the manuscript, and interpreting the findings.
DMA, RNK, NT, KK and AJA substantially contributed to the manuscript by providing review comments and edits. All authors have read and approved the final manuscript.

\section{Funding}

This study was funded by the UK General Medical Council (RMS 113361). The NIHR Greater Manchester Patient Safety Translational Research Centre (GMPS TRC-2012-1) funded the corresponding author's time spent in this project. MP is also a co-investigator in the Evidence Synthesis Working Group (project 390), which is supported by the NIHR School for Primary Care Research. The research team members were independent from the funding agencies. The views expressed in this manuscript are those of the authors and not necessarily those of the General Medical Council, the National Health Service, the NIHR or the Department of Health. The funders had no role in the design and conduct of the study; the collection, management, analysis, and interpretation of the data; and the preparation, review, or approval of the manuscript.

\section{Availability of data and materials}

Data and statistical code are available upon request from the corresponding author.

Ethics approval and consent to participate

Not applicable

\section{Consent for publication}

Not applicable

\section{Competing interests}

All authors have completed the ICMJE uniform disclosure form at wwW. icmje.org/coi_disclosure.pdf and all other authors declare no support from any organisation for the submitted work, no financial relationships with any organisations that might have an interest in the submitted work in the previous 3 years, and no other relationships or activities that could appear to have influenced the submitted work.

\section{Author details}

${ }^{1}$ National Institute for Health Research School for Primary Care Research, Centre for Primary Care and Health Services Research, Division of Population Health, Health Services Research and Primary Care, School of Health Sciences, Faculty of Biology, Medicine and Health, University of Manchester, Manchester Academic Health Science Centre, Williamson Building, Oxford Road, Manchester M13 9PL, UK. ${ }^{2}$ National Institute for HealthResearch Greater Manchester Patient Safety Translational Research Centre, School of Health Sciences, University of Manchester, Manchester M13 9PL, UK. ${ }^{3}$ Centre for Pharmacoepidemiology and Drug Safety, Division of Pharmacy and Optometry, University of Manchester, Manchester, UK. ${ }^{4}$ Pharmacy Department, Greater Manchester Mental Health NHS Foundation Trust University of Manchester, Manchester M25 3BL, UK. ${ }^{5}$ Division of Primary Care, School of Medicine, University of Nottingham, Nottingham NG7 2RD, UK.

Received: 12 June 2020 Accepted: 1 September 2020 Published online: 06 November 2020

\section{References}

1. Donaldson MS. An Overview of To Err is Human: Re-emphasizing the Message of Patient Safety. In: Hughes RG, editor. Patient safety and quality: an evidence-based handbook for nurses. Rockville: Agency for Healthcare Research and Quality (US); 2008. Chapter 3. Available from: https://www. ncbi.nlm.nih.gov/books/NBK2673/.

2. Stelfox HT, Palmisani S, Scurlock C, Orav EJ, Bates DW. The "To Err is Human" report and the patient safety literature. Qual Saf Health Care. 2006;15(3): $174-8$.

3. Vincent $C$, Neale G, Woloshynowych M. Adverse events in British hospitals: preliminary retrospective record review. Brit Med J. 2001;322(7285):517-9.

4. The third WHO Global Patient Safety Challenge: medication without harm. Patient safety. WHO. Last accessed on 10 Sept 2020. Available at: https:// www.who.int/patientsafety/medication-safety/en/.

5. Institute for Healthcare Improvement. National Steering Committee for Patient safety. A national strategy for reducing harm. Last accessed on 10 
Sept 2020. Available at: http://www.hi.org/Engage/Initiatives/NationalSteering-Committee-Patient-Safety/Pages/default.aspx.

6. Makary MA, Daniel M. Medical error-the third leading cause of death in the US. BMJ. 2016;353:12139.

7. Ferner RE, Aronson JK. Clarification of terminology in medication errors: definitions and classification. Drug Saf. 2006;29(11):1011-22.

8. Baker GR, Norton PG, Flintoft V, Blais R, Brown A, Cox J, et al. The Canadian Adverse Events Study: the incidence of adverse events among hospital patients in Canada. CMAJ Canad Med Assoc J. 2004;170(11):1678-86.

9. Elliott R, Camacho E, Campbell F, Jankovic D, Martyn St James M, Kaltenthaler E, Wong R, Sculpher M, Faria R, (2018). Prevalence and economic burden of medication errors in the NHS in England. Rapid evidence synthesis and economic analysis of the prevalence and burden of medication error in the UK. Policy Research Unit in Economic Evaluation of Health and Care Interventions. Universities of Sheffield and York. Available at: http://www.eepru.org.uk/prevalence-and-economic-burden-ofmedication-errors-in-the-nhs-in-england-2/. Last accessed on 10 Sept 2020.

10. Elliott RA, Camacho E, Jankovic D, et al. Economic analysis of the prevalence and clinical and economic burden of medication error in England. BMJ Qual Saf. 2020. https://doi.org/10.1136/bmjgs-2019-010206.

11. Resar RK, Rozich JD, Classen D. Methodology and rationale for the measurement of harm with trigger tools. Qual Saf Health Care. 2003; 12(suppl 2):ii39-45.

12. Brown C, Hofer T, Johal A, Thomson R, Nicholl J, Franklin BD, et al. An epistemology of patient safety research: a framework for study design and interpretation. Part 1. Conceptualising and developing interventions. Qual Saf Health Care. 2008;17(3):158-62.

13. Vincent $C$, et al. The measurement and monitoring of safety. London: The Health Foundation - Inspring Improvement; 2013.

14. Avery AJ, Rodgers S, Cantrill JA, Armstrong S, Cresswell K, Eden M, et al. A pharmacist-led information technology intervention for medication errors (PINCER): a multicentre, cluster randomised, controlled trial and costeffectiveness analysis. Lancet. 2012;379(9823):1310-9.

15. Medication Safety and Quality. High-risk medicines - A PINCH. New South Wales Government. Clinical Excellence Commission. Available at: http:// www.cec.health.nsw.gov.au/keep-patients-safe/medication-safety-andquality/high-risk-medicines/A-PINCH. Last Assessed 2 Mar 2020.

16. Avery A, Barber N, Ghaleb M, Franklin BD, Armstrong S, Crowe $\mathrm{S}$, et al. Investigating theprevalence and causes of prescribing errors in general practice: the PRACtICe study. London: General Medical Council; 2012. https://www.gmc-uk.org/-/media/about/investigatingtheprevalenceand causesofprescribingerrorsingeneral practicethepracticestudyreoprtmay 2012. pdf?la=en\&hash=62C1821CA5CCC5A4868B86A83FEDE14283686C29, Accessed 22 Mar 2019.

17. Anderson $O$, Davis $R$, Hanna GB, Vincent CA. Surgical adverse events: a systematic review. Am J Surg. 2013;206(2):253-62.

18. Lilford R, Stirling S, Maillard N. Citation classics in patient safety research: an invitation to contribute to an online bibliography. Qual Saf Health Care. 2006:15(5):311-3.

19. Aronson JK, Ferner RE. Clarification of terminology in drug safety. Drug Saf. 2005;28(10):851-70.

20. Borenstein M, Hedges LV, Higgins JP, Rothstein HR. A basic introduction to fixed-effect and random-effects models for meta-analysis. Res Synth Methods. 2010;1(2):97-111

21. Moore SJ, Jenkins AT, Poppe LB, Rowe EC, Eckel SF. Significant publications about the medication use process in 2012. J Pharm Pract. 2015;28(4):387-97.

22. Keers RN, Plácido M, Bennett K, Clayton K, Brown P, Ashcroft DM. What causes medication administration errors in a mental health hospital? A qualitative study with nursing staff. PLoS One. 2018;13(10):e0206233.

23. Miller JJ. The inverse of the Freeman - Tukey double arcsine transformation. Am Stat. 1978;32(4):138.

24. Schwarzer G, Chemaitelly H, Abu-Raddad LJ, Rücker G. Seriously misleading results using inverse of Freeman-Tukey double arcsine transformation in meta-analysis of single proportions. Res Synth Methods. 2019;10(3):476-83.

25. Agresti A, Coull B. Approximate is better than exact for interval estimation of binomial proportions. Am Stat. 1998;52:2.

26. Newcombe RG. Interval estimation for the difference between independent proportions: comparison of eleven methods. Stat Med. 1998;17(8):873-90.

27. Higgins JP, Thompson SG, Spiegelhalter DJ. A re-evaluation of randomeffects meta-analysis. J Royal Stat Soc Series A, (Statistics in Society). 2009; 172(1):137-59.
28. Hallas J, Harvald B, Gram LF, Grodum E, Brosen K, Haghfelt T, et al. Drug related hospital admissions: the role of definitions and intensity of data collection, and the possibility of prevention. J Intern Med. 1990;228(2):83-90

29. Schumock GT, Thornton JP. Focusing on the preventability of adverse drug reactions. Hosp Pharm. 1992;27(6):538.

30. Higgins JPT, Thompson SG, Deeks JJ, Altman DG. Measuring inconsistency in meta-analyses. Brit Med J. 2003;327(7414):557-60.

31. Higgins JP, Thompson SG. Quantifying heterogeneity in a meta-analysis. Stat Med. 2002;21(11):1539-58.

32. R Core Team. $R$ version 3.2.2: a language and environment for statistical computing. Vienna: R Foundation for Statistical Computing; 2019. URL http://www.R-project.org/.

33. Buckley MS, Erstad BL, Kopp BJ, Theodorou AA, Priestley G. Direct observation approach for detecting medication errors and adverse drug events in a pediatric intensive care unit. Pediatr Crit Care Med. 2007:8(2): $145-52$.

34. De Boer M, Boeker EB, Ramrattan MA, Kiewiet JJS, Dijkgraaf MGW, Boermeester MA, et al. Adverse drug events in surgical patients: an observational multicentre study. Int J Clin Pharm. 2013;35(5):744-52.

35. Hamilton H, Gallagher P, Ryan C, Byrne S, O'Mahony D. Potentially inappropriate medications defined by STOPP criteria and the risk of adverse drug events in older hospitalized patients. Arch Intern Med. 2011;171(11): 1013-9.

36. Jha AK, Kuperman GJ, Rittenberg E, Teich JM, Bates DW. Identifying hospital admissions due to adverse drug events using a computer-based monitor. Pharmacoepidem Dr S. 2001;10(2):113-9.

37. Lopez L, Weissman JS, Schneider EC, Weingart SN, Cohen AP, Epstein AM. Disclosure of hospital adverse events and its association with patients' ratings of the quality of care. Arch Intern Med. 2009;169(20):1888-94.

38. Morimoto T, Sakuma M, Matsui K, Kuramoto N, Toshiro J, Murakami J, et al. Incidence of adverse drug events and medication errors in Japan: the JADE study. J Gen Intern Med. 2011;26(2):148-53.

39. Al Damen L, Basheti I. Preventability analysis of adverse drug reactions in a Jordanian hospital: a prospective observational study. Int J Clin Pharm. 2019;41: 1599-610. https://doi.org/10.1007/s1 1096-019-00925-0.

40. Ayani N, Sakuma M, Morimoto T, Kikuchi T, Watanabe K, Narumoto J, et al. The epidemiology of adverse drug events and medication errors among psychiatric inpatients in Japan: the JADE study. BMC Psychiatry. 2016;16(1): 303.

41. Rothschild JM, Mann K, Keohane CA, Williams DH, Foskett C, Rosen SL, et al. Medication safety in a psychiatric hospital. Gen Hosp Psychiatry. 2007;29(2): $156-62$.

42. Kunac DL, Kennedy J, Austin N, Reith D. Incidence, preventability, and impact of adverse drug events (ADEs) and potential ADEs in hospitalized children in New Zealand: a prospective observational cohort study. Paediatr Drugs. 2009;11(2):153-60.

43. Ligi I, Arnaud F, Jouve E, Tardieu S, Sambuc R, Simeoni U. latrogenic events in admitted neonates: a prospective cohort study. Lancet. 2008;371(9610): 404-10.

44. Ithnin M, Latif Z, Syaiful A, Rani M, Nor AK. Incidence of adverse drug reactions in a paediatric ward of a Malaysian hospital: a prospective observational study. Trop J Pharm Res. 2018;17:1423-31.

45. Chan M, Nicklason F, Vial JH. Adverse drug events as a cause of hospital admission in the elderly. Intern Med J. 2001;31(4):199-205.

46. Farcas A, Bucsa C, Sinpetrean A, Leucuta D, Mogosan C, Dumitrascu D, et al. Preventability analysis of adverse drug reactions detected in two internal medicine departments in Romania. Int Emerg Med. 2014;9(2):187-93.

47. Franceschi M, Scarcelli C, Niro V, Seripa D, Pazienza AM, Pepe G, et al. Prevalence, clinical features and avoidability of adverse drug reactions as cause of admission to a geriatric unit: a prospective study of 1756 patients. Drug Saf. 2008;31(6):545-56.

48. Harugeri A, Parthasarathi G, Ramesh M, Guido S, Basavanagowdappa H. Frequency and nature of adverse drug reactions in elderly in-patients of two Indian medical college hospitals. J Postgrad Med. 2011;57(3):189-95.

49. Tangiisuran B, Davies JG, Wright JE, Rajkumar C. Adverse drug reactions in a population of hospitalized very elderly patients. Drug Aging. 2012;29(8):669-79.

50. Panagioti M, Khan K, Keers RN, Abuzour A, Phipps D, Kontopantelis E, et al. Prevalence, severity, and nature of preventable patient harm across medical care settings: systematic review and meta-analysis. BMJ. 2019;366:14185.

51. Wolfe D, Yazdi F, Kanji S, Burry L, Beck A, Butler C, et al. Incidence, causes, and consequences of preventable adverse drug reactions occurring in 
inpatients: a systematic review of systematic reviews. PLoS One. 2018;13(10): e0205426.

52. Parekh N, Ali K, Stevenson JM, Davies JG, Schiff R, Van der Cammen T, et al. Incidence and cost of medication harm in older adults following hospital discharge: a multicentre prospective study in the UK. Br J Clin Pharmacol. 2018;84(8):1789-97.

53. Rankin A, Cadogan CA, Patterson SM, et al. Interventions to improve the appropriate use of polypharmacy for older people. Cochrane Database Syst Rev.2018;9(9):CD008165. https://doi.org/10.1002/14651858.CD008165.pub4.

54. Scottish Government Polypharmacy Model of Care Group. Polypharmacy Guidance, Realistic Prescribing 3rd Edition, 2018. Scottish Government.

55. Stewart D, Mair A, Wilson M, Kardas P, Lewek P, Alonso A, et al. Guidance to manage inappropriate polypharmacy in older people: systematic review and future developments. Expert Opin Drug Saf. 2017;16(2):203-13.

56. Moyen E, Camiré E, Stelfox HT. Clinical review: medication errors in critical care. Crit Care. 2008;12(2):208.

57. Kreckler S, Catchpole KR, New SJ, Handa A, McCulloch PG. Quality and safety on an acute surgical ward: an exploratory cohort study of process and outcome. Ann Surg. 2009;250(6):1035-40.

58. Sheikh A, Panesar SS, Larizgoitia I, Bates DW, Donaldson LJ. Safer primary care for all: a global imperative. Lancet Glob Health. 2013;1(4):E182-E3.

59. Alshehri GH, Keers RN, Ashcroft DM. Frequency and nature of medication errors and adverse drug events in mental health hospitals: a systematic review. Drug Saf. 2017;40(10):871-86.

60. Rodgers, S., Salema, N., Waring, J., Armstrong, S., Mehta, R., Bell, B., ... On behalf of the Scaling Up PINCER Evaluation and Implementation Teams, . (2018). Improving medication safety in general practices in the East Midlands through the PINCER intervention: Scaling Up PINCER. The Health Foundation. Available at: https://nottingham-repository.worktribe.com/ output/1778784

61. Impact of electronic prescribing on patient safety in hospitals: implications for the UK. the Pharmaceutical Journal. A Royal Pharmaceuitcal Society publication. 5th May 2016. Available at: https://www.pharmaceutical-journal. com/research/review-article/impact-of-electronic-prescribing-on-patientsafety-in-hospitals-implications-for-the-uk/20201013.fullarticle. Last Accessed 2 Mar 2020.

62. Jeffries M, Keers RN, Phipps DL, Williams R, Brown B, Avery AJ, et al. Developing a learning health system: insights from a qualitative process evaluation of a pharmacist-led electronic audit and feedback intervention to improve medication safety in primary care. PLoS One. 2018;13(10):e0205419.

63. Pontefract SK, Hodson J, Slee A, Shah S, Girling AJ, Williams R, et al. Impact of a commercial order entry system on prescribing errors amenable to computerised decision support in the hospital setting: a prospective prepost study. BMJ Qual Saf. 2018;27(9):725-36.

64. Fox A, Pontefract S, Brown D, Portlock J, Coleman J. Developing consensus on hospital prescribing indicators of potential harm for infants and children. Br J Clin Pharmacol. 2016;82(2):451-60.

65. Thomas SK, McDowell SE, Hodson J, Nwulu U, Howard RL, Avery AJ, et al. Developing consensus on hospital prescribing indicators of potential harms amenable to decision support. Br J Clin Pharmacol. 2013;76(5):797-809.

66. NHS England and NHS Improvement. The NHS Patient Safety Strategy. Safer culture, safer systems, safer patients. July 2019. Available at: https:// improvement.nhs.uk/documents/5472/190708_Patient_Safety_Strategy_for_ website_v4.pdf. Last Accessed 2 Mar 2020.

67. Russell LM, Doggett J, Dawda P, Wells R. Patient safety - handover of care between primary and acute care. Policy review and analysis. Canberra: National Lead Clinicians Group, Australian Government Department of Health and Ageing; 2013.

68. Dean B, Schachter M, Vincent C, Barber N. Causes of prescribing errors in hospital inpatients: a prospective study. Lancet. 2002;359(9315):1373-8.

69. Dean B, Schachter M, Vincent C, Barber N. Prescribing errors in hospital inpatients: their incidence and clinical significance. Qual Saf Health Care. 2002;11(4):340-4.

70. Sutherland A, Ashcroft DM, Phipps DL. Exploring the human factors of prescribing errors in paediatric intensive care units. Arch Dis Child. 2019; 104(6):588-95.

71. The NHS Patient Safety Strategy: Safer culture, safer systems, safer patients. NHS. July 2019. Available at: https://improvement.nhs.uk/documents/5472/1 90708_Patient_Safety_Strategy_for_website_v4.pdf. Last accessed on 10 Sept 2020.
72. Sujan M and Cooke MW. Proactive Risk Monitoring in Healthcare (PRIMO): prerequisites for deployment in diverse settings and the impact on safety culture. 5th April 2014. Available at: https://www.health.org.uk/sites/default/ files/PRIMO_FinalReport.pdf. Accessed 30 July 2020.

73. Patient safety in developing and transitional countries. New insights from Africa and the Eastern Mediterranean. World Health Organisation - a world alliance for safer health care, better knowledge for safer care. Available at: https://www.who.int/patientsafety/research/emro_afro_report.pdf?ua=1. Last Accessed 31 Mar 2020.

74. Wilson R, Michel P, Olsen S, Gibberd R, Vincent C, El-Assady R, et al. Patient safety in developing countries: retrospective estimation of scale and nature of harm to patients in hospital. BMJ. 2012;344(7850):1-14.

75. Parekh N, Ali K, Davies JG, Stevenson JM, Banya W, Nyangoma S, et al. Medication-related harm in older adults following hospital discharge: development and validation of a prediction tool. BMJ Quality \&amp. Safety. 2020;29(2):142-53.

76. Jones KH, Laurie G, Stevens L, Dobbs C, Ford DV, Lea N. The other side of the coin: harm due to the non-use of health-related data. Int J Med Inform. 2017:97:43-51.

77. Nabhan M, Elraiyah T, Brown DR, Dilling J, LeBlanc A, Montori VM, et al. What is preventable harm in healthcare? A systematic review of definitions. BMC Health Serv Res. 2012:12:128.

78. Hayward RA, Hofer TP. Estimating hospital deaths due to medical errors: preventability is in the eye of the reviewer. JAMA. 2001;286(4):415-20.

79. Bhise $V$, Sittig DF, Vaghani $V$, Wei L, Baldwin J, Singh $H$. An electronic trigger based on care escalation to identify preventable adverse events in hospitalised patients. BMJ Qual Saf. 2018;27(3):241-6.

80. Pharmaceutical Care Network Europe. PCNE statement on medication review 2013. Available at : https://www.pcne.org/upload/files/150_201605 04_PCNE_MedRevtypes.pdf. Last Accessed 3 Mar 2020.

\section{Publisher's Note}

Springer Nature remains neutral with regard to jurisdictional claims in published maps and institutional affiliations.

\section{Ready to submit your research? Choose BMC and benefit from:}

- fast, convenient online submission

- thorough peer review by experienced researchers in your field

- rapid publication on acceptance

- support for research data, including large and complex data types

- gold Open Access which fosters wider collaboration and increased citations

- maximum visibility for your research: over $100 \mathrm{M}$ website views per year

At BMC, research is always in progress.

Learn more biomedcentral.com/submissions 